\title{
How much Ultrafiltration in CKD-5 Patients?
}

\section{Thomas Ryzlewicz}

WBR Dialysis Centre Group DTZ (Germany), Lana, Italy

"Corresponding author: Ryzlewicz T, Senior consultant, WBR Dialysis Centre Group DTZ (Germany), Via Bolzano 15/4, Lana/ttalia/Alto Adige, Alto Adige 39011, Italy, Tel: +491728366625; E-mail: thomas.ryzlewicz@web.de

Received date: August 17, 2016; Accepted date: September 19, 2016; Published date: September 27, 2016

Copyright: () 2016 Ryzlewicz T. This is an open-access article distributed under the terms of the Creative Commons Attribution License, which permits unrestricted use, distribution, and reproduction in any medium, provided the original author and source are credited

\section{State of the Art}

Along the decades of haemodialysis, there had many knowledge accumulated concerning the cardiovascular stability. Nursing compromises always the observation of the patients. This is you never come out. Today the number of patient one nurse has to treat had increase because of economic reasons. So there is much work to do for the nurses and the risk concerning a severe blood pressure had increased. All clinical points to assess to real dry weight are valid in the future. But in practice, this has difficulties: If you ask two different doctors concerning the dry weight of one single and individual patient, you definitely will get three answers...

Approaching to this theme, an overview of the different fluid compartments will be helpful. A $70 \mathrm{~kg}$ human has 401 total body water. $2 / 3$ of this $(>25 \mathrm{l})$ are the intra-cellular volume, $1 / 3(15 \mathrm{l})$ is the extracellular volume. Blood volume consists of both: The RBC's belong to the intra-cellular Volume, the serum compart. In case of an underfilling there happens a poor cardiovascular state with the danger of a blood pressure decrease (in worst case even a breakdown of the circulation) or muscle-cramps by too much ultrafiltration. Ultrafiltration is only reasonable when water (from over-hydration) is present. Cardio-vascular stability you will reach, when you prevent the under-filling ( $>$ the much reduced flow from the interstitial compartment into the serum compartment).

There are three different approaches to assess the fluid volume of a CKD-5 patient in order to prevent an under-filling:

1. By calculation

2. dBV-measurement (supra-sonic)

3. hydraulic assessment by the tunnelled dialysis catheter

Fluid assessment by calculation: If the dry weight seems correct, then a net ultrafiltration (referred to the scale) of $4 \%$ of the dry weight will be possible without any problems. In case of $6 \%$ ultrafiltration there will happen discomfort (>blood pressure decrease, muscle cramps), when you try to reach the real body weight. When you remains 0,5 or $1,0 \mathrm{~kg}$ over the correct dry weight, the treatment will have no symptoms. This method derived from KS [1].

\section{Fluid Assessment by dBV-Measurement}

The monitor is equipped with supra-sonic technique (transmitter and receiver) to measure continuously a kind of Haematocrit by Red Blood Cells of the blood. The result has expressed as the change of the blood volume during the actual ultrafiltration. A reduction of blood volume of to $-10 \%$ during ultrafiltration is a stable situation. When overriding this $-10 \%$ limit, then symptoms may appear. In this situation you can check the entire situation (patient's condition, blood pressure measurement) in order to make a decision concerning the ultrafiltration (continue or reduce?). A patient with hypertension will tolerate dBV's up to -15 or $-17 \%$. But the high percentages of dBV's are warnings to you. Perhaps you may install the infusion line for the case a sudden blood pressure decrease. In cases of severe over-hydration or left-heart deficiency, the $\mathrm{dBV}$ will run into a bigger positive region during high ultrafiltration rates. So you can continue the high-rate ultra-filtration, as there is a bigger fluid overload in these cases.

\section{Fluid Assessment by Hydraulic Measurement by a Tunnelled Catheter}

Tunnelled catheters are by far more a problem as a help in dialysis centres (> see DOPPS report). But they are often used, especially in the US. If these are present, you can get information concerning the individual hydration state of the patient. In the ICU's, the central venous pressure is a classical method to assess the hydraulic state of a patient [2-4]. There is no big difference for this assessment of body fluid between a central venous ICU catheter (tip in the vena cava superior) and a tunnelled dialysis catheter (tip in the right atrium of the heart). The patients will set into a straight horizontal position. Because of general hygienic conditions the lengthening of the catheter (according to Twardowski冈 his lengthening-set-up for the CAPD) will used. In the end, the clamp will open to see in which direction the saline fluid and in what extend will run than the patient will connected with the bloodline.

\section{General Thoughts and Critics}

At first, you should check, whether the committed dry weight has really realized in the last time. A question concerning perhaps happened problems is reasonable. Now and then, a planned dry weight will realized with profiling of ultrafiltration and/or conductivity. In these cases, it seems that the real dry weight is too low. One word to the profiling (ultrafiltration and conductivity): A descending ultrafiltration profile can be helpful. A descending conductivity profile along the dialysis never can be useful, as this is an amplifying to the actual reached ultrafiltration. The automatic profiling (ultrafiltration and conductivity) according to the dBV (Haemo-Control): At first, nursing has to set-up clinical input of e.g. 13 dialysis into the system. The system tries to reach the dry weight. If this is not realistic, alarms will follow and in the end a lengthening of the treatment time (> problems of organisation will follow). The better option is, to drive the ultrafiltration with the results of the dBV measurement by hand. To discuss the paper of Ciccone MM et al. (> Project Leonardo, Vascular Health and Risk Management 2010:6 297-305): Whenever health managers had worked very successful in order to improve the patient's self-care facilities together with his general practitioners, the diagnosis of end-stage renal disease had been excluded in this study. The dialysis nurse remains the primary to assess and to handle the water compartment of her patient. Dialysis does tap the cardio-vascular circulation system, so in case of overriding the hydraulic equilibrium, 
Page 2 of 2

there is the general risk of under-filling of blood volume in the RRT With elevated relation between one dialysis-nurse to e.g. six or seven patients, this is a very responsible work. Every support (in this way: technical, by calculations of hydraulic or by one assistant) will be helpful. A health manager cannot really help in the dialysis treatment.

\section{Conclusion}

All the three approaches will contribute to prevent the danger of under-filling. So they are helpful to the nursing, an improvement of the therapy concerning the volume state of the patient will reached. DOPPS report had clearly pointed out, that longer survival had reached by the prevention of severe blood pressure decreases.

\section{References}

1. Schaefer $\mathrm{K}$ personal commitment concerning "Fluid assessment by calculation".

2. Demers HG, Siebold G, Geier J, Hoeffler D (1986) Dialyse ohne Shunt: Silikonkatheter im rechten Vorhof - Nieren- und Hochdruckkrankheiten 15: $460-461$.

3. Marik R, Baram M, Bobbak V (2008) Does Central Venous Pressure Predict Fluid Responsiveness? Chest 134: 172-178.

4. Horl W "Blut-Volumen-Messung" in his book "Dialyseverfahren in Klinik und Praxis", Horl and Wanner (eds.,) Thieme publishers. 